\title{
Deformations of Quantum Hyperplanes
}

\author{
GERHARD POST \\ Department of Applied Mathematcs, University of Twente. PO Box 217, \\ 7500 AE Enschede, The Netheriands
}

(Received: 27 January 1995)

\begin{abstract}
We consider the quantum hyperplane $x^{2} x^{j}=q_{1}, x^{3} x^{1}(i, j=1 . . n)$ and define and consider

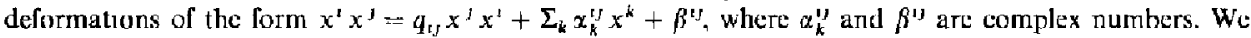
prove that for generic $q_{1,}$ no nontrivial deformations exist for $n \geqslant 3$
\end{abstract}

Mathematics Subject Classifications (1991). 17H 3̧7, 16E40.

Key words: quantum hyperplanes, PBW theorem, deformations, cohomology

\section{Introduction}

One of the main objects in the study of quadratic algebras is the PoincareBirkhoff-Witt (PBW) theorem. For the case of (colour) Lie algebras g (see, e.g., [1]) this theorem provides us with a basis of the universal enveloping algebra $U(\mathfrak{g})$ of $g$. It is well known that in this case the PBW theorem is equivalent to Jacobi's identity.

In this Letter we study certain quadratic algebras, namely those of which the pure quadratic part is a quantum hyperplane, $x^{i} x^{j}=q_{i j} x^{j} x^{i}$. The quantum hyperplane is an (Abelian) colour algebra is, hence, satisfies the PBW theorem (Proposition 2.2). We study deformations of the form $x^{i} x^{j}=q_{i j} x^{j} x^{i}+\Sigma_{k} \alpha_{k}^{i j} x^{k}+\beta^{i j}$, where $\alpha_{k}^{i j}$ and $\beta^{i j}$ are complex numbers. However, we require that the deformations still satisfy the PBW theorem. This will give a set of constraints on $\alpha_{k}^{i j}$ and $\beta^{i j}$, which resemble conditions occurring in formal deformation theory, see, e.g.,[2]. The technique we use is the Diamond lemma [3]. It turns out that (in case we take $\beta^{\prime \prime}=0$ ) we have two types of constraints: first linear ones, which are trivially satisfied in the case of Lie algebras, and secondly quadratic constraints. These last constraints reduce to Jacobi's identity in the case of Lie algebras.

Our technique resembles that of [4], but the approach is more or less transversal: our constraints emerge from the requirement of $\mathrm{PBW}$, whereas in [4] $\mathrm{PBW}$ is satisfied, and the requirement of associativity leads to the constraints. The cohomology in [4] is Hochschild cohomology, whereas our cohomology is related to cohomology of the underlying linear space, as in Lie algebra theory; in particular for low dimensions the cohomology is easy to compute.

The organization of the Letter is as follows. First we define the cohomology for a quantum hyperplane, and then we study a class of deformations, which we define, as 
said above, in terms of the PBW theorem. We find necessary and sufficient conditions in explicit form. Using this result, we prove that the generic quantum hyperplane is 'rigid'. We discuss as examples the cases $n=2$, and $n=3$ with $q_{12}=q_{23}$.

\section{Quantum Hyperplane and Cohomology}

2.1. Let $V$ be an $n$-dimensional linear space with basis $X=\left\{x^{1}, \cdots, x^{n}\right\}$, and let $T(V)$ be the tensor algebra of $V$.

DEFINITION 2.1. $\mathscr{A}_{q}$ is called the $n$-dimensional quantum hyperplane if $\mathscr{A}_{q}=T(V) / I_{q}$ where $I_{q}$ is the ideal generated by

$$
x^{i} x^{i}-q\left(x^{i}, x^{j}\right) x^{j} x^{i}
$$

with $q: X \times X \rightarrow \mathbb{C}^{*}$ satisfies $q\left(x^{i}, x^{j}\right) q\left(x^{j}, x^{i}\right)=1$.

It is obvious that $\mathscr{A}_{q}$ is $\mathbb{Z}^{n}$-graded; if $|\cdot|$ denotes the degree, we can take $\left|x^{i}\right|=e_{i}=(0, \cdots, 0,1,0, \cdots, 0)$. We extend $q$ to a mapping on all monomials of $T(V)$ in the following way:

$$
q\left(x^{i_{1}} \cdots x^{i_{s}}, x^{j_{1}} \cdots x^{j_{r}}\right)=\prod_{\substack{i \in\left\{i_{1}, \cdots, i_{s}\right\} \\ j \in\left\{j_{1}, j_{r}\right\}}} q\left(x^{i}, x^{j}\right) .
$$

Doing this we have that in $\mathscr{A}_{q}$ holds

$$
x^{i_{1}} \cdots x^{i_{s}} x^{j_{1}} \cdots x^{j_{r}}=q\left(x^{i_{1}} \cdots x^{i_{s}}, x^{j_{1}} \cdots x^{j_{r}}\right) x^{j_{1}} \cdots x^{j_{r}} x^{i_{1}} \cdots x^{i_{s}} .
$$

Usually we write $q_{i j}$ instead of $q\left(x^{i}, x^{j}\right)$. From the requirement $q_{i j} q_{j i}=1$, we have that $q_{i i} q_{i i}=1$. Throughout we will assume that $q_{i i}=1$ for convenience of presentation; hence the relation $x^{i} x^{i}-q\left(x^{i}, x^{i}\right) x^{i} x^{i}$ is trivial.

PROPOSITION 2.2. Let $\mathscr{A}_{q}$ be a quantum hyperplane with generators $\left\{x^{1}, \cdots x^{n}\right\}$. Then the monomials

$$
x^{i_{1}} x^{i_{2}} \cdots x^{i_{s}} \quad i_{1} \geqslant i_{2} \cdots \geqslant i_{s}, \quad s=0,1,2, \ldots
$$

form a (linear) basis of $\mathscr{A}_{q^{*}}$

Proof. (Well-known); it is a special case of Proposition 3.3.

The basis in Proposition 1.2 we will call the Poincaré-Birkhoff-Witt basis.

2.2. We will define three cohomology complexes, a left, right and a bicomplex for a quantum hyperplane. To do so we introduce the notion of a module. By definition a left (right) module will be left (right) representation of the associative algebra with unit $\mathscr{A}_{q}$. Moreover a bimodule $M$ will be a left representation $\rho$ on $M$ and a right representation $\sigma$ on $M$ such that $(\rho(x) m) \sigma(y)=\rho(x)(m \sigma(y))$ for all $x, y \in \mathscr{A}_{q}, m \in M$. If we say module, it can be either left, right or bi. 
DEFINITION 2.3. Let $M$ be an $\mathscr{A}_{q}$-module. The $M$-valued skew-symmetric forms $\alpha$ of order $p$ is the space of multi-linear mappings

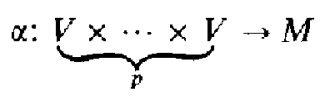

such that

$$
\alpha\left(\cdots, x^{i}, x^{j}, \cdots\right)=-q_{i j} \alpha\left(\cdots, x^{j}, x^{i}, \cdots\right) .
$$

This space we denote by $C^{p}(V ; M)$.

We define $C^{p}(V ; M)=0$ for $p<0$. Moreover, $C^{0}(V ; M)$ can be naturally identified with $M$ by the map $\phi: C^{0}(V ; M) \rightarrow M ; \phi(\alpha)=\chi(1)$.

DEFINITION 2.4. Let $\mathscr{A}_{q}$ be a quantum hyperplane with generators $x^{1}, \cdots x^{n}$, and $V=\left\langle x^{1}, \cdots, x^{n}\right\rangle$.

(a) For a left module $M_{\ell}$ we define $\mathrm{d}_{\ell}: C^{p}\left(V ; M_{i}\right) \rightarrow C^{p+1}\left(V ; M_{\ell}\right)$ by

$$
\mathrm{d}_{f} \alpha\left(x^{i_{0}}, \cdots, x^{i_{p}}\right)=\sum_{s=0}^{p}(-1)^{s} q\left(x^{i_{0}} \cdots x^{i_{s}-1}, x^{i_{s}}\right) x^{i_{s}} \alpha\left(x^{i_{0}}, \cdots, \widehat{x_{s}}, \cdots x^{i_{p}}\right)
$$

(b) for a right module $M_{r}$ we define $\mathrm{d}_{r}: C^{p}\left(V ; M_{r}\right) \rightarrow C^{p+1}\left(V ; M_{r}\right)$ by

$$
\mathrm{d}_{r} \alpha\left(x^{u_{u}}, \cdots, x^{i_{p}}\right)=\sum_{s=0}^{p}(-1)^{s} q\left(x^{i_{s}}, x^{i_{s}+1} \cdots x^{i_{p}}\right) \alpha\left(x^{i_{0}}, \cdots, \widehat{i_{s}}, \cdots x^{i_{p}}\right) x^{i_{s}}
$$

(c) for a bimodule $M$ we define d: $C^{p}(V ; M) \rightarrow C^{p+1}(V ; M)$ by

$$
\mathrm{d} \alpha=\mathrm{d}_{\rho} \alpha-\mathrm{d}_{\mathrm{r}} \alpha \text {. }
$$

It is easily checked that $d_{\ell}, d_{r}$ and $d$ are well-defined, and that the images are indeed in $C^{p+1}$. The definitions of $\mathrm{d}_{\ell}$ coincides with what the definition for a colour Lie algebra and a left-module $M$ should be (for a colour-commutative algebra $\alpha_{4}$ ). We leave it to the reader to check:

PROPOSITION 2.5. Notations as in Definition 2.4. Then $\mathrm{d}_{\rho}^{2}=0, \mathrm{~d}_{\mathrm{r}}^{2}=0$, $\mathrm{d}_{l} \mathrm{~d}_{\mathrm{r}}=-\mathrm{d}_{\mathrm{r}} \mathrm{d}_{\text {, and }} \mathrm{d}^{2}=0$.

\section{Deformations of $\mathscr{A}_{q}$}

Let $V$ be a linear space as before, $\left\{x^{1}, \cdots, x^{n}\right\}$ a basis of $V$ and $\mathscr{N}_{q}$ the quantum hyperplane. Define $\mathscr{A}_{4, t}$ to be $T(V) / I_{q, l}$, where $I_{q, t}$ is generated by

$$
x^{i} x^{j}-q_{i j} x^{i} x^{i}-t \sum_{s} \alpha_{s}^{i j} x^{s}-t^{2} \beta^{i j}
$$

Here $t, \alpha_{s}^{1 J}, \beta^{i j}$ are complex numbers. By construction, $\mathscr{A}_{q, t}$ is an associative algebra with unit, and could therefore be called a deformation of $\mathscr{A}_{q}$. It is easy to prove that the tensors of Proposition 2.2

$$
x^{i_{1}} x^{i_{2}} \cdots x^{i_{s}}, \quad i_{1} \geqslant i_{2} \cdots \geqslant i_{s}
$$


generate $\mathscr{A}_{q, t}$. It is not at all obvious, and in general not true, that these are a basis in $\mathscr{A}_{q, t}$. This observation will be is the motivation for our definition.

DEFINITION 3.1. Let the notations be as above. The algebra $\mathscr{A}_{q, t}=T(V) / I_{q, t}$ will be called a deformation of $\mathscr{A}_{q}$ if the elements

$$
x^{i_{1}} x^{i_{2}} \cdots x^{i_{s}}, \quad i_{1} \geqslant i_{2} \cdots \geqslant i_{s}
$$

form a basis of $\mathscr{A}_{q, 1}$.

Note that the situation as sketched here is not formal: we consider $t \in \mathbb{C}$ and not a formal letter. For all $t \in \mathbb{C}^{*}, \mathscr{A}_{q, t}$ and $\mathscr{A}_{q, 1}$ are isomorphic as unital algebras. The isomorphism is given by $x^{i} \rightarrow t x^{i}$.

LEMMA 3.2. Let $\mathscr{A}_{q, 1}$ as above be a deformation of $\mathscr{A}_{q}$. Define

$$
\alpha: V \times V \rightarrow V \text { and } \beta: V \times V \rightarrow \mathbb{C}
$$

by

$$
\alpha\left(x^{i}, x^{j}\right)=\sum_{s} \alpha_{s}^{i j} x^{s} \text { and } \beta\left(x^{i}, x^{j}\right)=\beta^{i j} .
$$

Then $\alpha \in C^{2}(V ; V)$ and $\beta \in C^{2}(V ; C)$.

Proof. We consider $\mathscr{A}_{q}$ as a left and right module over itself in the standard way. By associativity of $\mathscr{A}_{q}$, we have that $\mathscr{A}_{q}$ is a bimodule. It remains to show that $\alpha$ and $\beta$ are skew-symmetric. We have

$$
x^{i} x^{j}=q_{i j} x^{j} x^{i}+t \alpha\left(x^{i}, x^{j}\right)+t^{2} \beta\left(x^{i}, x^{j}\right) .
$$

Multiplying with $q_{j i}$, we find

$$
x^{j} x^{i}=q_{j i} x^{i} x^{j}-t q_{j i} \alpha\left(x^{i}, x^{j}\right)-t^{2} q_{j i} \beta\left(x^{i}, x^{j}\right) .
$$

It follows that $x\left(x^{j}, x^{i}\right)=-q_{j i} \alpha\left(x^{i}, x^{j}\right)$ and $\beta\left(x^{j}, x^{i}\right)=-q_{j i} \beta\left(x^{i}, x^{j}\right)$, since otherwise there would be a linear relation between $x^{1}, \cdots, x^{n}$ and 1 .

Note that when we say $\alpha \in C^{2}(V ; V)$ we mean $\alpha \in C^{2}\left(V ; \alpha_{q}\right)$ with $\alpha\left(x^{i}, x^{j}\right) \in V$. In particular, we have that $\mathrm{d} \alpha \in C^{3}\left(V ; \not \mathscr{F}_{q}\right)$. Similarly with $\beta$; one sees that $\mathrm{d} \beta\left(x^{i}, x^{j}\right) \in C^{3}(V ; V)$.

To describe the necessary and sufficient conditions on $\alpha$ and $\beta$ to define a deformation, we need an extra operation from $C^{2}\left(V ; \mathscr{A}_{q}\right) \times C^{2}(V ; V)$ to $C^{3}\left(V ; \mathscr{A}_{q}\right)$, cf. [5]. Let $\beta, \gamma \in C^{2}\left(V ; \alpha A_{q}\right)$ and $\alpha \in C^{2}(V ; V)$. Then we define

$$
\gamma_{-}\left(x^{i}, x^{j}\right)= \begin{cases}0, & i \geqslant j, \\ \gamma\left(x^{i}, x^{j}\right), & i<j .\end{cases}
$$

Moreover, for $i<j<k$, we define

$$
\begin{aligned}
\beta * \alpha & \left(x^{i}, x^{j}, x^{k}\right) \\
= & \beta_{+}\left(\alpha\left(x^{i}, x^{j}\right), x^{k}\right)-q_{j k} \beta_{+}\left(\alpha\left(x^{i}, x^{k}\right), x^{j}\right)+q_{i j} q_{i k} \beta_{+}\left(\alpha\left(x^{j}, x^{k}\right), x^{i}\right)- \\
& -\beta_{+}\left(x^{i}, \alpha\left(x^{j}, x^{k}\right)\right)+q_{i j} \beta_{+}\left(x^{j}, \alpha\left(x^{i}, x^{k}\right)\right)-q_{j k} q_{i k} \beta_{+}\left(x^{k}, \alpha\left(x^{i}, x^{j}\right)\right)
\end{aligned}
$$


and extend $\beta * x$ to be in $C^{3}\left(V ; \mathscr{A}_{q}\right)$ by skew-symmetry. It will turn out that these products play a role in the obstructions.

PROPOSITION 3.3. Let $\mathscr{A}_{q}, \mathscr{A}_{q, t}$ and $\alpha \in C^{2}(V ; V), \beta \in C^{2}(V ; C)$ be as above. Then $\mathscr{A}_{4, t}$ is a deformation of $\mathscr{A}_{q}$ if and only if

(a) $\mathrm{d} \alpha=0$,

(b) $\mathrm{d} \beta=\alpha * \alpha$,

(c) $\beta * \alpha=0$.

Proof. To investigate the basis of $\mathscr{A}_{q . t}$ we use the Diamond Lemma [3]. For this we have to start with $x^{i} x^{j} x^{k}(i<j<k)$ and rewrite it in normal form (i.e. expressed in monomials with decreasing indices) in two different ways; by first rewriting $x^{i} x^{j}$ and by first rewriting $x^{j} \mathrm{x}^{k}$. We have

$$
\begin{aligned}
\left(x^{i} x^{j}\right) x^{k}= & \left(q_{i j} x^{j} x^{i}+t \alpha\left(x^{i}, x^{j}\right)+t^{2} \beta\left(x^{i}, x^{j}\right)\right) x^{k} \\
= & q_{i j}\left(q_{i k} x^{j} x^{k} x^{i}+t x^{j} \alpha\left(x^{i}, x^{k}\right)+t^{2} x^{j} \beta\left(x^{i}, x^{k}\right)\right)+ \\
& +t \alpha\left(x^{i}, x^{j}\right) x^{k}+t^{2} \beta\left(x^{i}, x^{j}\right) x^{k} \\
= & q_{i j} q_{i k}\left(q_{j k} x^{k} x^{j} x^{i}+t \alpha\left(x^{j}, x^{k}\right) x^{i}+t^{2} \beta\left(x^{j}, x^{k}\right) x^{i}\right)+ \\
& +q_{i j} t x^{j} \alpha\left(x^{i}, x^{k}\right)+q_{i j} t^{2} \beta\left(x^{i}, x^{k}\right)+t \alpha\left(x^{i}, x^{j}\right) x^{k}+t^{2} \beta\left(x^{i}, x^{j}\right) x^{k} .
\end{aligned}
$$

Similarly rewriting $x^{i}\left(x^{i} x^{k}\right)$ yields

$$
\begin{aligned}
x^{i}\left(x^{j} x^{k}\right)= & q_{i j} q_{i k} q_{j k} x^{k} x^{j} x^{i}+t x^{i} \alpha\left(x^{j}, x^{k}\right)+t^{2} x^{i} \beta\left(x^{j}, x^{k}\right)+ \\
& +q_{j k} t x\left(x^{i}, x^{k}\right) x^{j}+q_{j k} t^{2} \beta\left(x^{i}, x^{k}\right) x^{j}+q_{j k} q_{i k} t x^{k} \alpha\left(x^{\prime}, x^{j}\right)+ \\
& +q_{j k} q_{i k} t^{2} x^{k} \beta\left(x^{i}, x^{j}\right) .
\end{aligned}
$$

These two results are not yet in normal form. Let us consider them in more detail. First note that the cubic terms cancel (this proves Proposition 2.2). Further, we have quadratic terms. These quadratic terms are also not yet in normal form: they are of the form $x^{\prime \prime} \alpha(\cdot, \cdot \cdot)$ and $\alpha(\cdot, \cdot \cdot) x^{*}$. Reducing these will give quadratic terms, linear terms and constant terms (in $x$ ). For the moment, we are only interested in quadratic terms. Hence rewriting $x^{\prime \prime} \alpha(\cdot, \cdot \cdot)$ and $\alpha(\cdot, \cdot) x^{-}$is just interchanging $x^{r}$ and $x^{s}$ with the proper $q_{s}$; in other words at this moment we can consider these terms to be in $\mathscr{A}_{4}$ instead of $\mathscr{A}_{4, t}$. So we need

$$
\begin{aligned}
& x^{i} \alpha\left(x^{j}, x^{k}\right)+q_{j k} \alpha\left(x^{i}, x^{k}\right) x^{j}+q_{j k} q_{i k} x^{k} \alpha\left(x^{i}, x^{j}\right) \\
& \quad=q_{i j} q_{i k} \alpha\left(x^{j}, x^{k}\right) x^{i}+q_{i j} x^{i} \alpha\left(x^{i}, x^{k}\right)+\alpha\left(x^{i}, x^{j}\right) x^{k} \text { in } \mathscr{A}_{q}
\end{aligned}
$$

Or

$$
\operatorname{da}\left(x^{i}, x^{j}, x^{k}\right)=0 \text { for } i<j<k,
$$

and by skew-symmetry of $\alpha$ we find $\mathrm{d} \alpha=0$.

Let us proceed to the linear terms. These are terms of the forms $x^{\prime \prime} \beta(\cdot, \cdot), \beta(\cdot, \cdot) x^{\prime \prime}$ (these give rise to $\mathrm{d} \beta$ ), but also $x^{\prime \prime} \alpha(\cdot, \cdot)$ and $\alpha(\cdot, \cdot) x^{\prime \prime}$ give 
rise to linear terms; for instance

$$
\begin{aligned}
t x^{i} \alpha\left(x^{j}, x^{k}\right)= & \sum_{s} x^{i} t \alpha_{s}^{j k} x^{s} \\
= & \sum_{s \leqslant i} t \alpha_{s}^{j k} x^{i} x^{s}+\sum_{s>i} q_{i s} t \alpha_{s}^{j k} x^{s} x^{i}+ \\
& +\sum_{s>i, r} t^{2} \alpha_{s}^{j k} \alpha_{r}^{i s} x^{r}+\sum_{s>i} t^{3} \alpha_{s}^{j k} \beta^{i s}
\end{aligned}
$$

Carefully comparing all terms linear in $x$ yields:

$$
\mathrm{d} \beta\left(x^{i}, x^{j}, x^{k}\right)=\alpha * \alpha\left(x^{i}, x^{j}, x^{k}\right) \quad(i<j<k),
$$

and by skew-symmetric we find $\mathrm{d} \beta=\alpha * \alpha$.

Similarly the constant terms yield $\beta * \alpha=0$.

We remark that this result shows that we are in a situation similar to the one that one encounters in formal deformation theory: infinitesimal there is a linear constraint $d \alpha=0$, and the higher term (in $t$ ) give polynomial obstructions. Indeed, we also have that infinitesimal the deformation is trivial if $\alpha$ is a coboundary.

PROPOSITION 3.4. Let $\mathscr{A}_{q, t}$ be as before. If $\alpha=d \gamma$, then there exist new coordinates $\bar{x}^{i}=x^{i}-t \gamma\left(x^{i}\right)$ such that $\mathscr{A}_{q, t}$ is given by

$$
\bar{x}^{i} \bar{x}^{j}=\bar{x}^{j} \bar{x}^{i}+t^{2} \delta\left(x^{i}, x^{j}\right) \text {, with } \delta \in C^{2}\left(V ; \alpha_{q}\right)
$$

Proof. We denote by $\doteq$ equality modulo $t^{2}$. Then

$$
\begin{aligned}
\bar{x}^{i} \bar{x}^{i} & =\left(x^{i}-t \gamma\left(x^{i}\right)\right)\left(x^{j}-t \gamma\left(x^{j}\right)\right) \\
& \doteq x^{i} x^{j}-t\left(x^{i} \gamma\left(x^{j}\right)+\gamma\left(x^{i}\right) x^{j}\right) \\
& \doteq q_{i j} x^{i} x^{i}+t\left(\mathrm{~d} \gamma\left(x^{i}, x^{j}\right)-x^{i} \gamma\left(x^{j}\right)-\gamma\left(x^{i}\right) x^{j}\right) \\
& \doteq q_{i j}\left(\bar{x}^{j}+t \gamma\left(x^{j}\right)\right)\left(\bar{x}^{i}+t \gamma\left(x^{i}\right)\right)+t\left(-q_{i j} x^{j} \gamma\left(x^{i}\right)-q_{i j} \gamma\left(x^{j}\right) x^{i}\right) \\
& \doteq q_{i j} \bar{x}^{j} \bar{x}^{i} .
\end{aligned}
$$

EXAMPLE 3.5. Let us consider the case $n=2$. We will write $x=x_{1}$ and $y=x_{2}$. The quantum plane $\mathscr{A}_{q}$ is given by $x y=q y x$, and we consider $\mathscr{A}_{q, 1}$ given by

$$
x y=q y x+t\left(\alpha_{1} x+\alpha_{2} y\right)+t^{2} \beta
$$

For the corresponding 2-forms $\alpha$ and $\beta$, we have $\mathrm{d} \alpha=0$ and $\mathrm{d} \beta=0$ automatically, as $\mathrm{d} \alpha, \mathrm{d} \beta \in C^{3}\left(V ; \mathscr{A}_{q}\right)$ and $\operatorname{dim}(V)=2$. For $\gamma \in C^{1}(V ; \mathbb{C})$ we have

$$
\mathrm{d} \gamma(x, y)=x \gamma(y)-q y \gamma(x)-q \gamma(y) x+\gamma(x) y
$$

or $\mathrm{d} \gamma(x, y)=\gamma_{2}(1-q) x+\gamma_{1}(1-q) y$, where $\gamma_{i}=\gamma\left(x^{i}\right)$. By taking $\gamma_{1}=\alpha_{2} /(1-q)$ and $\gamma_{2}=\alpha_{1} /(1-q)$, we have that $\mathrm{d} \gamma=\alpha(q \neq 1)$. Hence, $H^{2}(V ; V)=0(q \neq 1)$. It follows that up to a change of coordinates, all deformations of the quantum plane are isomorphic to the $q$-Weyl algebra $x y=q y x+\beta$. 


\section{Rigidity of the Generic Quantum Hyperplane}

We have seen that for $n=2$ there is (generically) exactly one deformation of the quantum plane, corresponding to the fact that $H^{2}(V ; V)=0$ and $\operatorname{dim}\left(H^{2}(V ; \mathrm{C})\right)=1$ in this case. We will prove that this is an exception. For $n \geqslant 3$, we have that $H^{2}(V ; C)=0$ if for all $i \neq j$ there is a $k$ (different from $i$ and $j$ ) such that $q_{i k} q_{j k} \neq 1$. Indeed, if $\beta \in C^{2}(V ; \mathbb{C})$, then

$$
\begin{aligned}
\mathrm{d} \beta( & \left(x^{i}, x^{j}, x^{k}\right) \\
= & \left(1-q_{i j} q_{i k}\right) \beta\left(x^{j}, x^{k}\right) x^{i}+\left(q_{j k}-q_{i j}\right) \beta\left(x^{i}, x^{k}\right) x^{j}+ \\
& +\left(q_{i k} q_{j k}-1\right) \beta\left(x^{i}, x^{j}\right) x^{k} .
\end{aligned}
$$

From which it is clear that $\beta\left(x^{\mathrm{l}}, x^{j}\right)=0$. Moreover, we have

PROPOSITION 4.1. Let $\mathscr{A}_{q}$ be a quantum hyperplane with $q_{i j} \neq 1$ and $q_{i k} q_{j k} \neq 1$ for all mutually different $i, j, k$. Then $H^{2}(V ; V)=0$.

Proof. (By induction, $n=2$ is treated above). Suppose $\alpha \in C^{2}(V ; V)$ and $\mathrm{d} \alpha=0$. Let

$$
\alpha\left(x^{1}, x^{j}\right)=\sum_{s} \alpha_{s}^{i j} x^{s}
$$

Writing out $\mathrm{d} \alpha\left(x^{i}, x^{j}, x^{k}\right)=0$ yields

$$
\begin{aligned}
& x^{i} \alpha\left(x^{j}, x^{k}\right)-q_{i j} x^{j} \alpha\left(x^{i}, x^{k}\right)+q_{i k} q_{j k} x^{k} \alpha\left(x^{i}, x^{j}\right)- \\
& \quad-q_{i j} q_{i k} x\left(x^{j}, x^{k}\right) x^{i}+q_{j k} \alpha\left(x^{i}, x^{k}\right) x^{j}-\alpha\left(x^{i}, x^{j}\right) x^{k}=0
\end{aligned}
$$

Now it is clear that the coefficient of $x^{k} x^{k}$ above is $\left(q_{i k} q_{j k}-1\right) \alpha_{k}^{i j}$. Hence, we have that $\alpha_{k}^{i j}=0$ for $k \neq i$ and $k \neq j$.

Now we can restrict $\alpha$ to $\bar{V}=\left\langle x^{1}, \ldots, x^{n-1}\right\rangle$. This restriction we denote by $\bar{\alpha}$. From $\alpha_{k}^{i j}=0$ for $k \neq i$ and $k \neq j$ it follows that $\bar{\alpha} \in C^{2}(\bar{V} ; \bar{V})$, and $\mathrm{d} \alpha=0$ implies $\overline{\mathrm{d}} \bar{\alpha}=0$. By induction we can find an element $\bar{\beta}$ such that $\overline{\mathrm{d}} \bar{\beta}=\bar{\alpha}$. We extend $\bar{\beta}$ to $\beta \in C^{\prime}(V ; C)$ by defining $\beta\left(x^{n}\right)=\alpha_{1}^{n} /\left(1-q_{1_{n}}\right)$. We prove that $\mathrm{d} \beta-\alpha$. For this we need to prove that $\hat{\alpha}=x-\mathrm{d} \beta=0$. Let

$$
\tilde{\alpha}\left(x^{i}, x^{j}\right)=\sum_{s} \tilde{\alpha}_{s}^{i j} x^{s}
$$

From our construction, we know that $\tilde{\alpha}_{k}^{i j}=0$ for $i, j<n$ and $\tilde{\alpha}_{1}^{1 n}=0$ (since $\left.\beta\left(x^{n}\right)=\alpha_{1}^{1 n} /\left(1-q_{1_{n}}\right)\right)$. Since also $\mathrm{d} \tilde{\alpha}=0$, it remains to prove that $\tilde{\alpha}_{j}^{j n}=0$ and $\tilde{\alpha}_{n}^{j n}=0$ $(j<n)$.

Look at $\mathrm{d} \tilde{\alpha}\left(x^{i}, x^{j}, x^{n}\right)=0$. The coefficient of $x^{i} x^{n}$ gives $\tilde{\alpha}_{n}^{j n}\left(1-q_{i j}\right)=0$, hence $\tilde{x}_{n}^{j n}=0$ for all $j=1, \cdots, n-1$. Finally taking $\mathrm{d} \tilde{\alpha}\left(x^{1}, x^{j}, x^{n}\right)=0$, and looking at the coefficient of $x^{1} x^{j}$, we find $\tilde{\alpha}_{j}^{j m}\left(1-q_{1 n}\right)=0$. Hence, we have $\tilde{x}=0$ as we wished to prove.

EXAMPLE 4.2. Let us consider the casc $n=3$, with $q_{i j} \neq 1$. From the previous proposition we need to take $q_{i k} q_{j k}=1$ to find something interesting. Let us take 
$q_{12}=q$ and $q_{23}=q$ and $q_{13} \neq q^{-1}$. One can easily calculate that $\operatorname{dim}\left(H^{2}(V ; V)\right)=1$ and $\operatorname{dim}\left(H^{2}(V ; \mathbb{C})\right)=1$. The most general solution for a deformation is

$$
\begin{aligned}
& x^{1} x^{2}=q x^{2} x^{1}+t \alpha_{1}^{12} x^{2}+t \alpha_{2}^{12} x^{2}+\frac{t^{2}}{q-1} \alpha_{2}^{12} \alpha_{1}^{12}, \\
& x^{1} x^{3}=q_{13} x^{3} x^{1}+t \alpha_{1}^{13} x^{1}+t \alpha_{2}^{13} x^{2}+t \frac{q_{13}-1}{q-1} \alpha_{2}^{12} x^{3}+t^{2} \beta^{13}, \\
& x^{2} x^{3}=q x^{3} x^{2}+t \frac{q-1}{q_{13}-1} \alpha_{1}^{13} x^{2}+t \alpha_{1}^{12} x^{3}+\frac{t^{2}}{q_{13}-1} \alpha_{1}^{13} \alpha_{1}^{12} .
\end{aligned}
$$

This contains Witten's second deformation of su(2) as a special case, cf. [6] and [7]. By changing basis, i.e. by transforming with a $\gamma \in C^{1}(V ; \mathbb{C})$, we can assume $\alpha_{1}^{12}=\alpha_{2}^{12}=\alpha_{1}^{13}=0$. In that case the only term that changes (compared to the quantum hyperplane) is

$$
x^{1} x^{3}=q_{13} x^{3} x^{1}+t \alpha_{2}^{13} x^{2}+t^{2} \beta^{13}
$$

\section{References}

1. Bahhturin, Y. A., Mikhalev, A. A., Petrogradsky, V. M. and Zaicev, M. V., Infinite Dimersional Lie Algebras, Walter de Gruyter, Berlin, 1992.

2. Gerstenhaber, M. and Shack, S. D., Algebraic cohomology and deformation theory, in Deformation Theory of Algebras and Structure and Application, NATO ASI series C-Vol, 247, Kluwer, Dordrecht, 1992.

3. Bergman, G. M., The diamond lemma for ring theory, Adv. Math. 29 (1978), 178-218

4. Braverman, A. and Gaitsgory, D., Poincaré-Birkhof-Witt theorem for quadratic algebras of Koszul type, Preprint hep-th/9411113.

5. Nijenhuis, A. and Richardson, R. W., Deformations of Lie algebra structures, J. Math. Mech. 17 (1967), $89 \rightarrow 105$.

6. Witten, E., Gauge theories, vector models and quantum groups, Nuclear Phys. B 330 (1990), 285-346.

7. Faitly, D., Quantum deformations of SU(2), J. Phys. A 23 (1990), 183-187. 